\title{
Exploring the impact of EGFR T790M neighboring SNPs on ARMS-based T790M mutation assay
}

\author{
SANPENG XU, YAQI DUAN, LIPING LOU, FENGJUAN TANG, JUAN SHOU and GUOPING WANG \\ Institute of Pathology, Tongji Hospital, Tongji Medical College, \\ Huazhong University of Science and Technology, Wuhan, Hubei 430030, P.R. China
}

Received May 29, 2015; Accepted August 5, 2016

DOI: $10.3892 / \mathrm{ol} .2016 .5184$

\begin{abstract}
The present study aimed to explore the influence of T790M neighboring single nucleotide polymorphism (SNP) on the sensitivity of amplification refractory mutation system (ARMS)-based T790M mutation assay. Three ARMS-quantitative polymerase chain reaction (qPCR) systems (system 1 had a forward ARMS primer without rs1050171, system 2 included a forward ARMS primer with rs1050171 and system 3 contained the above two forward ARMS primers) were used to detect the T790M mutation in two series plasmid samples and genomic DNA (gDNA) of the cell line H1975. A total of 670 formalin-fixed paraffin-embedded (FFPE) tumor samples from non-small cell lung cancer patients were used to detect the epidermal growth factor receptor (EGFR) gene T790M mutation by direct sequencing and ARMS-qPCR. The ARMS-qPCR system 1 effectively detected samples with as low as 1\% T790M mutant plasmid 1 (without rs1050171) and with 50\% T790M mutant plasmid 2 (with rs1050171), while the ARMS-qPCR system 2 detected samples with 20 and $50 \%$ T790M mutant plasmid 1, in addition to samples with 1\% T790M mutant plasmid 2. For the ARMS-qPCR system 3, samples with as low as $1 \%$ T790M mutant plasmids 1 or 2 were effectively detected. For gDNA analysis of the cell line H1975, the T790M mutation was effectively detected by the ARMS-qPCR systems 2 and 3 ( $\sim 50 \%$ mutation rate), but was detected with a low mutation abundance by the ARMS-qPCR system 1 ( $1 \%$ mutation rate). Of the 670 FFPE samples,
\end{abstract}

Correspondence to: Dr Guoping Wang, Institute of Pathology, Tongji Hospital, Tongji Medical College, Huazhong University of Science and Technology, 1095 Jiefang Da Dao, Wuhan, Hubei 430030, P.R. China

E-mail: wanggp@hotmail.com

Abbreviations: NSCLC, non-small cell lung cancer; EGFR T790M, epidermal growth factor receptor gene T790M mutation; ARMS, amplification refractory mutation system; SNP, single nucleotide polymorphism

Key words: non-small cell lung cancer, epidermal growth factor receptor gene T790M mutation, amplification refractory mutation system, single nucleotide polymorphism, rs1050171
5 cases were identified to have the T790M mutation by sequencing and by the ARMS-qPCR system 1. One sample (named N067), which was considered as T790M-negative by sequencing, was demonstrated to have the T790M mutation using the ARMS-qPCR system 1. Sample N094, which was variant homozygous for rs1050171 and was indicated to be T790M-negative by sequencing and by the ARMS-qPCR system 1, was identified to have the T790M mutation with the ARMS-qPCR system 3. The A-variant allele frequency of rs 1050171 was observed to be $28.2 \%$ in the 670 FFPE tumor samples, while the presence of rs148188503 (c. C2355T, p. T785T) was observed in sample N558, and a novel SNP with a base substitution (c. T2375C) at position 792 (p. L792P) in exon 20 of the EGFR gene was observed in sample N310. rs1050171 is a high-frequency SNP located near T790M, and the mutation statuses of rs1050171 appear to influence the sensitivity of the ARMS-based T790M detection system, thus generating a $14.3 \%$ false-negative rate $(1 / 7)$. The present study proposes the risk that target neighboring SNPs (as far as 8 bp away in the present study) may exert on the sensitivity of ARMS-based detection methods.

\section{Introduction}

The use of reversible epidermal growth factor receptor (EGFR) tyrosine kinase inhibitors (TKIs), including gefitinib and erlotinib, which function by competitively binding at the adenosine triphosphate-binding cleft of the receptor kinase domain of EGFR and consequently blocking the kinase activation and subsequent downstream signal transduction processes, produces secondary resistance in the majority of lung cancer patients (1). Previous studies have demonstrated that the majority of the acquired resistance is due to the EGFR T790M mutation (2-6). Second-generation irreversible EGFR TKIs, including BIBW2992 (afatinib) and PF-002999804, were developed to solve the drug resistance problem, but did not yield the desired results due to their narrow therapeutic window (7-9). The third-generation T790M specific inhibitors, mainly AZD9291 and CO-1686, are currently under clinical trials in patients with acquired resistance $(7,8)$.

Formalin-fixed paraffin-embedded (FFPE) samples have been widely recognized as common clinical materials for EGFR mutation detection, in which DNA may be partially degraded (10). EGFR mutation is a heterogeneous somatic 
mutation whose abundance may vary widely (11). Therefore, it is important to select a highly sensitive detection method for these low quality DNA samples and for those with low mutation abundances.

The EGFR T790M mutation may originate from small subclonal populations in the primary tumor, and may become dominant later on during EGFR-TKIs treatment (12). Early detection of the T790M mutation is of significance to aid the clinician to adjust the treatment timely, so that the patient can obtain the most effective therapy while reducing drug cost and waste. To date, the amplification refractory mutation system (ARMS) has been widely used in clinical gene mutation detection, including $E G F R, K R A S, B R A F$ and phosphatidylinositol-4,5-bisphosphate 3-kinase, catalytic subunit alpha (PIK3CA), due to its high sensitivity (13-24).

The cell line H1975 is EGFR T790M-positive ( 50\% mutation rate), but in our attempt to develop a T790M ARMS-based quantitative polymerase chain reaction (qPCR) method, the cell line H1975 was detected as T790M-positive with low mutation abundance $(\sim 1 \%$ mutation rate) using the ARMS-qPCR method (referred to as the ARMS-qPCR system 1), which was validated as having a detection limit of $1 \%$ in our preliminary study. The ARMS-qPCR method combines the ARMS primer and a unique fluorescent probe molecule, with the mutant allele selectively amplified by ARMS, and the amplified PCR product sensitively and specifically detected by the fluorescent probe system (24). Subsequent sequencing data revealed that the cell line H1975 is also a variant homozygous for rs1050171 (also designated as c. G2361A or p. Q787Q). We hypothesized that rs1050171 may affect the sensitivity of the ARMS method, thereby producing false-negative results. By searching the National Center for Biotechnology Information (NCBI) single nucleotide polymorphism (SNP) database, numerous T790M neighboring SNPs were identified, which were located within the ARMS primer design range (http://www.ncbi.nlm.nih. gov/projects/SNP/snp_ref.cgi?rs=121434569). The majority of them had no frequency data, with the exception of rs1050171, which has a high variant allele mutation rate (16.2\%) in the Chinese population. These SNPs may influence the detection sensitivity of the ARMS-based T790M mutation detection assay, thus carrying the risk of wrong interpretation.

Since the sensitive detection of the T790M mutation is very important for the individualized therapy of non-small cell lung cancer (NSCLC) patients (25), any factors affecting the sensitivity of the detection method should be a matter of concern. The present study aimed to investigate whether rs1050171 affects the detection sensitivity of the ARMS-based T790M mutation assay, as well as to determine the frequency of rs1050171 in NSCLC patients, and to identify the frequency of any other SNPs possibly neighboring T790M in NSCLC patients.

\section{Materials and methods}

Cell line. The H1975 [American Type Culture Collection (ATCC) ${ }^{\circledR}$ CRL-5908D ${ }^{\text {TM }}$ ] andHT29 (ATCC ${ }^{\circledR} \mathrm{HTB}^{-38^{\mathrm{TM}}}$ ) human tumor cell lines were purchased from the ATCC (Manassas, VA, USA) in December 2013. The cell lines were grown in standard conditions and validated by genotyping for EGFR. The genotypes of the cell lines exactly matched those described in the
Catalog of Somatic Mutations in Cancer (http://cancer.sanger. ac.uk/cosmic/sample/overview?id=1513408).

Development of three T790M ARMS-qPCR systems. The T790M ARMS-qPCR system 1 contained a forward ARMS primer (named T790M-F1, without rs1050171) designed with a mismatch at the penultimate nucleotide position of the mutation site to specifically amplify the T790M mutant allele, a reverse primer and a minor groove binder probe labeled with the fluorescein amidite (FAM) fluorescent dye. In the T790M ARMS-qPCR system 2, the primer T790M-F1 was replaced by T790M-F2 (with rs1050171). In the T790M ARMS-qPCR system 3, both T790M-F1 and T790M-F2 primers were included. A reference (RF) reaction system was designed in the EGFR gene to measure the quantity of both T790M-negative and T790M mutant alleles, so that the ratio of mutant to wild-type sequence could be measured. The primers and probes were synthesized by Invitrogen (Thermo Fisher Scientific, Inc., Waltham, MA, USA) and Applied Biosystems (Thermo Fisher Scientific, Inc.), respectively. The 5X PCR buffer, deoxynucleotides and Taq polymerase were purchased from Takara Bio Inc. (Otsu, Japan). The primers and probes are listed in Table I, and the schematic diagram of the three T790M ARMS-PCR systems is represented in Fig. 1. The reactions were performed in $25-\mu 1$ volumes containing $5 \mu 15 \mathrm{X}$ PCR buffer, $200 \mu \mathrm{M}$ dNTPs, $1 \mu \mathrm{l}$ forward and reverse primers (10 $\mu \mathrm{M}$ each), $1 \mu \mathrm{l}$ probe $(5 \mu \mathrm{M}), 2 \mu \mathrm{l}$ template DNA (adjusted to $10 \mathrm{ng} / \mu \mathrm{l}$ ) and $0.1 \mu \mathrm{l}$ Taq polymerase $(5 \mathrm{U} / \mu \mathrm{l})$. qPCR was conducted using a 7500 Real-Time PCR System (Applied Biosystems; Thermo Fisher Scientific, Inc.) under the following conditions: Initial denaturation at $95^{\circ} \mathrm{C}$ for $5 \mathrm{~min}$, followed by 40 cycles of $95^{\circ} \mathrm{C}$ for $15 \mathrm{sec}$ and $60^{\circ} \mathrm{C}$ for $1 \mathrm{~min}$, with fluorescence FAM reading at $60^{\circ} \mathrm{C}$ of each cycle. The quantification cycle $(\mathrm{Cq})$ represents the threshold at which the signal was detected above the background fluorescence (25). The $\Delta \mathrm{Cq}$ value was calculated as the difference between the $\mathrm{T} 790 \mathrm{M}$ mutation $\mathrm{Cq}$ and the RF Cq.

Comparison of the three ARMS-qPCR systems to detect T790M mutation in mixed samples with or without rs 1050171 and in the cell line H1975. The T790M mutant plasmid 1 (without rs1050171) was developed and quantified according to Zhang et al (25). The T790M mutant plasmid 2 (with rs1050171) was developed as follows: A DNA fragment containing the T790M mutation and rs1050171 was amplified from the genomic DNA (gDNA) of the cell line H1975 using primers T790M-F2 and T790M-R. The PCR cycling conditions were $94^{\circ} \mathrm{C}$ for $5 \mathrm{~min}$; followed by 40 cycles at $94^{\circ} \mathrm{C}$ for $30 \mathrm{sec}, 55^{\circ} \mathrm{C}$ for $30 \mathrm{sec}$ and $72^{\circ} \mathrm{C}$ for $1 \mathrm{~min}$; and a final extension at $72^{\circ} \mathrm{C}$ for $10 \mathrm{~min}$. The PCR product was then ligated into the pEASY-T1 vector (Beijing TransGen Biotech Co., Ltd., Beijing, China), and DNA sequencing was used to verify the accuracy of the fragment. Different amounts of T790M mutant plasmids 1 or 2 were mixed with $20 \mathrm{ng}$ wild-type EGFR HT29 gDNA to yield $1,2,5,10,20$ and $50 \%$ mutation rates, respectively. The two series mixed samples and the gDNA of the cell line H1975 were used to explore the sensitivity of the three ARMS-qPCR systems, and the detection results were compared to assess the influence of rs1050171 on the effective detection of T790M. 
A

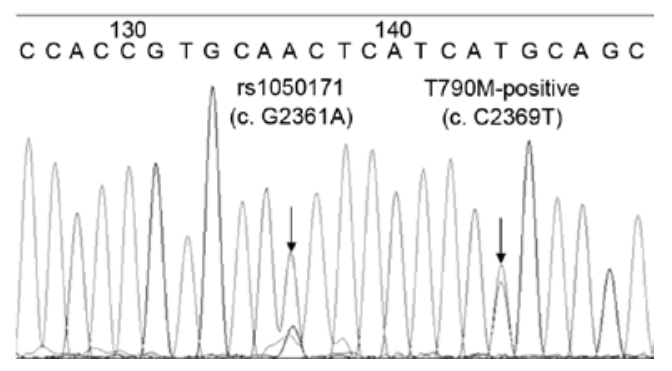

C

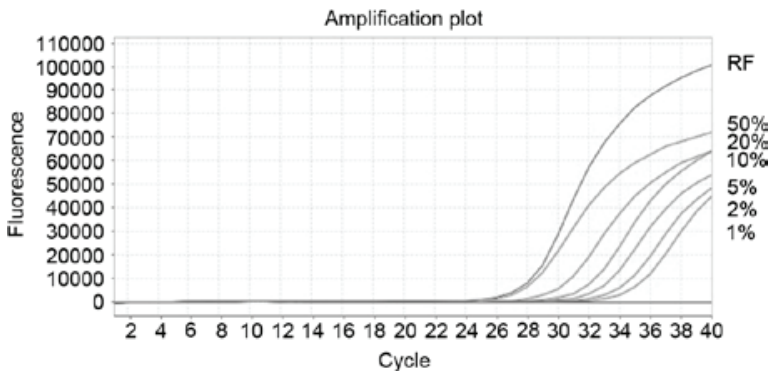

E

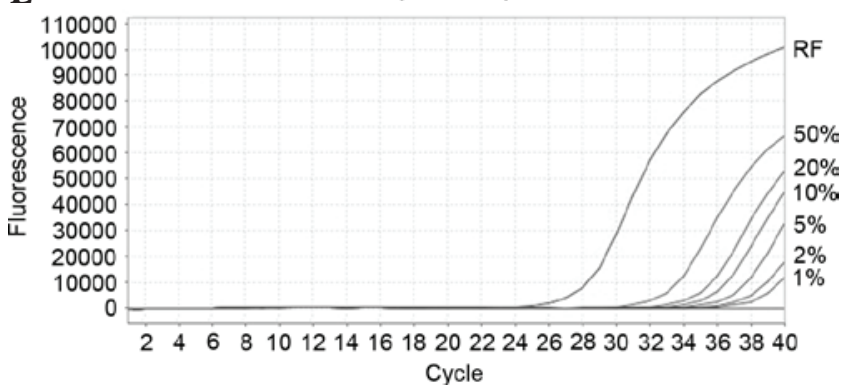

G

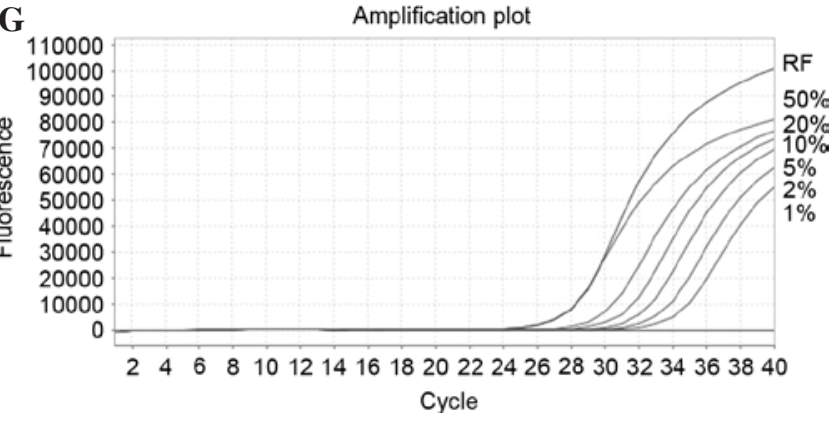

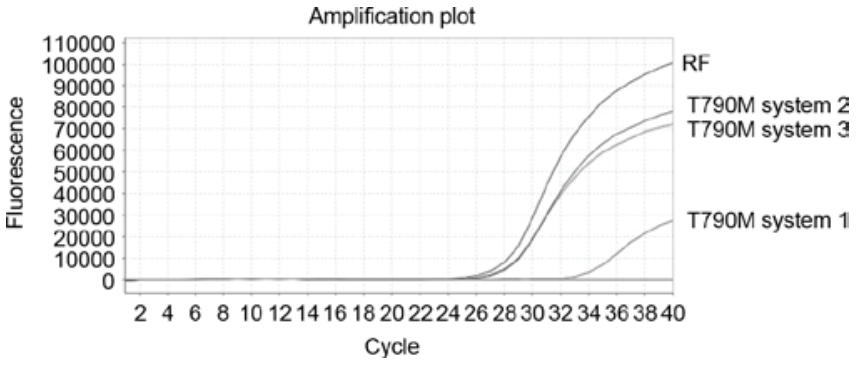

D

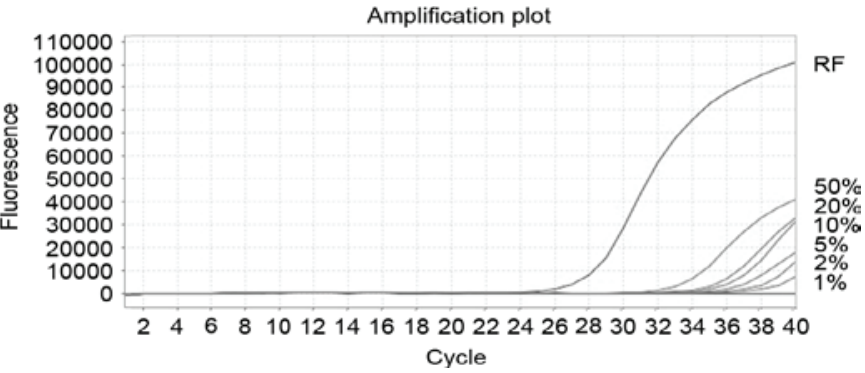

F

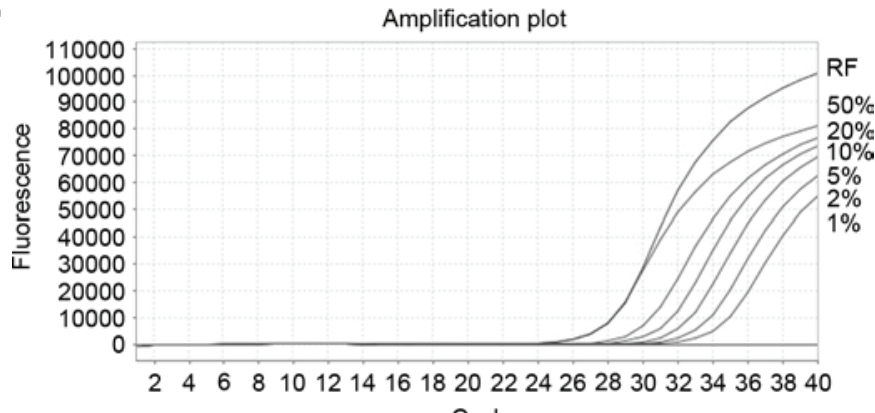

H

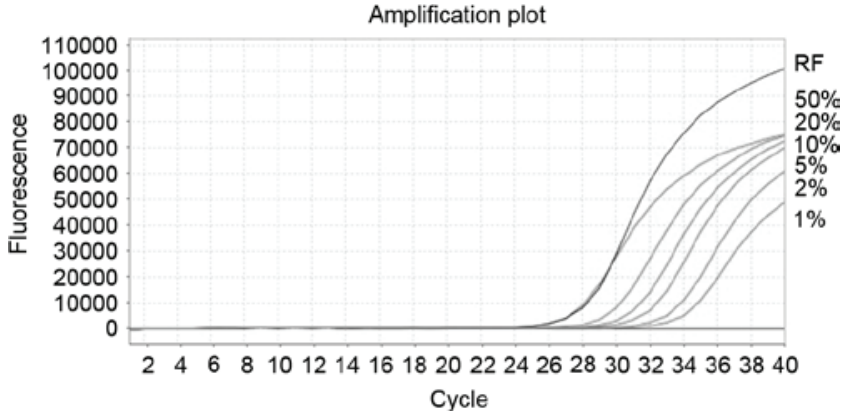

Figure 2. Detection results of cell line H1975 and mixed samples by the three T790M ARMS-qPCR systems. (A) The cell line H1975 has an apparent T790M mutation ( $\sim 50 \%$ mutation rate) and is homozygous mutant genotype of rs1050171. The nucleotide location of the single nucleotide polymorphism is indicated by the arrows ( $\downarrow$ ). (B) The cell line H1975 was detected to have a low abundance of the T790M mutation by the ARMS-qPCR system 1 ( $\sim 1 \%$ mutation rate), but was identified to have an obvious T790M mutation by the ARMS-qPCR systems 2 and 3 ( $\sim 50 \%$ mutation rate). (C) Samples with 1-50\% T790M mutant plasmid 1 could be detected by the ARMS-qPCR system 1. (D) Samples with 50\% T790M mutant plasmid 2 could be detected by the ARMS-qPCR system 1 , but not those with 1-20\% T790M mutant plasmid 2. (E) Samples with 20 and 50\% T790M mutant plasmid 1 could be detected by the ARMS-qPCR system 2, but not those with 1-10\% mutant plasmid 1. (F) Samples with 1-50\% T790M mutant plasmid 2 could be detected by the ARMS-qPCR system 2. (G) Samples with 1-50\% T790M mutant plasmid 1 could be detected by the ARMS-qPCR system 3. (H) Samples with 1-50\% T790M mutant plasmid 2 could be detected by the ARMS-qPCR system 3. ARMS, amplification refractory mutation system; qPCR, quantitative polymerase chain reaction; RF, reference.

(Fig. 3A). In the sample N310, a novel SNP with a base substitution (c. T2375C) in the EGFR gene (exon 20, position 792, p. L792P) was observed, which was not included in the SNP database of NCBI (Fig. 3B). In total, 5 cases (0.75\%) appeared to have the T790M mutation. Detailed information regarding the mutation frequencies of T790M neighboring SNPs in the 670 FFPE samples analyzed is presented in Table II.

T790M mutation detection results by the different ARMS- $q P C R$ systems. Of the 670 cases, 6 cases $(0.9 \%)$ appeared to have the T790M mutation by the T790M ARMS-qPCR system 1 .
Sample N067, which was firstly determined as T790M-negative by sequencing, it was identified to be T790M-positive by the ARMS-qPCR system 1. Of the 19 samples with T790M mutation, or with $T 790 \mathrm{M}$ amplification but with $\Delta \mathrm{Cq}$ values above the cut-off value of 7.5 and which were homozygous mutant genotype of rs1050171, the T790M ARMS-qPCR system 3 was used to detect the T790M mutation. As expected, sample N094 was determined to have the T790M mutation (Fig. 4).

Comparison of the T790M mutation detection results by different methods and the corresponding rs1050171 mutation 
Table II. Mutation frequencies of T790M neighboring SNPs in 670 formalin-fixed paraffin-embedded samples.

\begin{tabular}{|c|c|c|c|c|c|c|c|c|c|}
\hline \multirow[b]{2}{*}{ SNPs } & \multicolumn{3}{|c|}{ rs1050171 } & \multicolumn{3}{|c|}{ rs148188503 } & \multicolumn{3}{|c|}{ c. T2375C, p. L792P } \\
\hline & $\mathrm{G} / \mathrm{G}$ & $\mathrm{G} / \mathrm{A}$ & $\mathrm{A} / \mathrm{A}$ & $\mathrm{C} / \mathrm{C}$ & $\mathrm{C} / \mathrm{T}$ & $\mathrm{T} / \mathrm{T}$ & $\mathrm{T} / \mathrm{T}$ & $\mathrm{T} / \mathrm{C}$ & $\mathrm{C} / \mathrm{C}$ \\
\hline $\begin{array}{l}\text { Cases, } \mathrm{n} \\
(\%)\end{array}$ & $\begin{array}{c}316 \\
(47.20)\end{array}$ & $\begin{array}{c}330 \\
(49.20)\end{array}$ & $\begin{array}{c}24 \\
(3.60)\end{array}$ & $\begin{array}{c}669 \\
(99.85)\end{array}$ & $\begin{array}{c}0 \\
(0.00)\end{array}$ & $\begin{array}{c}1 \\
(0.15)\end{array}$ & $\begin{array}{c}669 \\
(99.85)\end{array}$ & $\begin{array}{c}1 \\
(0.15)\end{array}$ & $\begin{array}{c}0 \\
(0.00)\end{array}$ \\
\hline
\end{tabular}

SNP, single nucleotide polymorphism.

Table III. Detection results of various samples with the T790M mutation using different methods.

\begin{tabular}{|c|c|c|c|c|c|c|}
\hline \multirow[b]{2}{*}{ Sample name } & \multirow[b]{2}{*}{ Gender } & \multirow[b]{2}{*}{ Pathology } & \multicolumn{2}{|c|}{ Sequencing } & \multirow{2}{*}{$\begin{array}{c}\text { T790M by the } \\
\text { ARMS-qPCR system } 1\end{array}$} & \multirow{2}{*}{$\begin{array}{l}\text { T790M by the } \\
\text { ARMS-qPCR system } 3\end{array}$} \\
\hline & & & rs1050171 & T790M & & \\
\hline N019 & Female & $\mathrm{ADC}$ & $\mathrm{G} / \mathrm{G}$ & Positive & Positive & Positive \\
\hline N060 & Female & $\mathrm{ADC}$ & $\mathrm{G} / \mathrm{G}$ & Positive & Positive & Positive \\
\hline N067 & Female & $\mathrm{ADC}$ & $\mathrm{G} / \mathrm{A}$ & Negative & Positive & Positive \\
\hline N072 & Female & $\mathrm{ADC}$ & $\mathrm{G} / \mathrm{G}$ & Positive & Positive & Positive \\
\hline N333 & Male & $\mathrm{ADC}$ & $\mathrm{G} / \mathrm{A}$ & Positive & Positive & Positive \\
\hline N512 & Male & $\mathrm{ADC}$ & $\mathrm{G} / \mathrm{A}$ & Positive & Positive & Positive \\
\hline N094 & Female & $\mathrm{ADC}$ & $\mathrm{A} / \mathrm{A}$ & Negative & Negative & Positive \\
\hline
\end{tabular}

ADC, adenocarcinoma; ARMS, amplification refractory mutation system; qPCR, quantitative polymerase chain reaction.

analysis. Of the 670 FFPE samples analyzed, 5 cases were detected as having the $\mathrm{T} 790 \mathrm{M}$ mutation by sequencing, and 6 cases were identified as having the T790M mutation by the ARMS-qPCR system 1 method. The consistency of the two methods was $99.85 \%$ (669/670). Sample N094, which is variant homozygous for rs1050171, was firstly detected as T790M-negative by the T790M ARMS-qPCR system 1, but was confirmed to be T790M-positive by the T790M ARMS-qPCR system 3 . Of the 7 FFPE samples with the T790M mutation, 3, 3 and 1 cases were wild-type, heterozygous mutant and homozygous mutant genotypes of rs1050171, respectively. Detailed results of these analyses are presented in Table III.

\section{Discussion}

Three T790M ARMS-qPCR systems were developed in the present study: i) The ARMS-qPCR system 1 (without rs1050171 in the forward ARMS primer), which effectively detected samples with as low as $1 \%$ T790M mutant plasmid 1 (without rs1050171) and with 50\% T790M mutant plasmid 2 (with rs1050171); ii) the ARMS-qPCR system 2 (with rs1050171 in the forward ARMS primer), which detected samples with $20-50 \%$ T790M mutant plasmid 1 and with $1 \%$ T790M mutant plasmid 2; and iii) the ARMS-qPCR system 3 (where the two forward ARMS primers mentioned above were included), which effectively detected samples with as low as 1\% T790M mutant plasmids 1 or 2. For the cell line H1975, the ARMS-qPCR system 1 detected the T790M mutation with low abundance ( $\sim 1 \%$ mutation rate), and the ARMS-qPCR systems 2 and 3 effectively detected it with high abundance
( $\sim 50 \%$ mutation rate, consistent with the sequencing results). These results preliminarily indicated that the existence of rs1050171 affects the sensitivity of the ARMS-qPCR system used in the present study. To further confirm this result, 670 FFPE samples from NSCLC patients were used to detect the T790M mutation by sequencing and by the ARMS-qPCR system 1, and partial samples were detected by the ARMS-qPCR system 3. In the 670 FFPE samples, the variant-A allele frequency of rs1050171 was $28.2 \%$, with the wild-type, heterozygous and homozygous mutant genotype accounting for $47.2,49.2$ and $3.6 \%$, respectively. In addition, the results of 669 cases obtained by sequencing and by the ARMS-qPCR system 1 were consistent (99.85\%). Sample N067, which was firstly identified as T790M-negative and rs1050171 wild-type by sequencing, was identified as exhibiting the T790M mutation by the ARMS-qPCR systems 1 and 3, thus illustrating the higher sensitivity of the ARMS-qPCR method compared with direct sequencing. Notably, sample N094, which was detected as T790M-negative by sequencing and by the ARMS-qPCR system 1, was determined to have the T790M mutation by the ARMS-qPCR system 3. These results illustrate that rs1050171 actually influences the sensitivity of the ARMS-based T790M mutation detection method, particularly in samples with low abundance of T790M mutation.

A large number of patients who are resistant to EGFR-TKIs often harbor a pre-existing T790M EGFR mutation at very low levels within the original tumor population, which leads to resistance following treatment (12). Screening patients for low levels of T790M EGFR mutation prior to administering EGFR-TKIs treatment may be useful to assess the possibility 
A

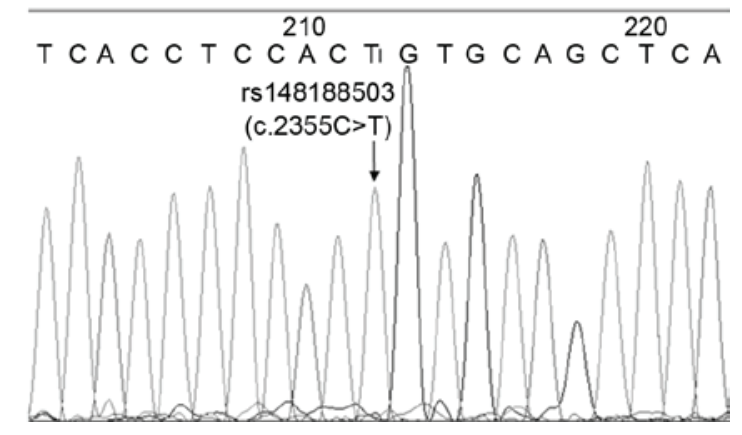

B

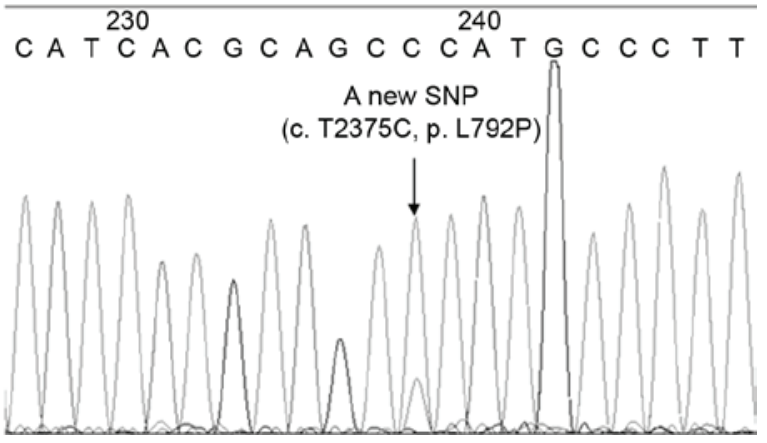

Figure 3. Sequencing results of samples (A) N558 and (B) N310. (A) Sample N558 was observed to be homozygous mutant genotype of rs148188503. (B) A new SNP was detected in sample N310. The nucleotide location of the SNP is indicated by the arrows $(\downarrow)$. SNP, single nucleotide polymorphism.

of disease relapse. Furthermore, monitoring the presence of T790M mutation in plasma during EGFR-TKIs treatment may be useful for future clinical decision making. In order to detect these low-level T790M variations in the primary tumor or their progression in plasma, it is important to apply reliable and sensitive mutation detection methods (12). With a detection limit of $1 \%$, the ARMS method is a sensitive, convenient and economic approach that is widely used in point mutation detection, and any factor affecting the sensitivity of this method should be addressed (24). According to the SNP database of NCBI, rs1050171 is an SNP with high mutation frequency located 8 bp prior to T790M. Under the influence of rs1050171, the T790M mutation in the gDNA of the cell line H1975 and in the FFPE sample N094 could not be effectively detected in the present study. Of the 670 FFPE samples analyzed, 6 and 7 cases were identified to have the T790M mutation by the ARMS-qPCR system 1 and 3, respectively, and the false-negative rate (1/7) was $14.3 \%$. Considering the significance of early detection of low-abundance T790M mutation, the false-negative rate resulted from rs1050171 should not be underestimated.

Among the 7 FFPE samples with the T790M mutation, $3(3 / 7,42.9 \%), 3(3 / 7,42.9 \%)$ and 1 cases $(1 / 7,14.3 \%)$ were wild-type, heterozygous and homozygous mutant genotype of rs1050171, respectively. Of the 316 wild-type, 330 heterozygous and 24 variant homozygous cases for rs1050171, 3 (3/316,0.95\%), $3(3 / 330,0.91 \%)$ and $1(1 / 24,4.17 \%)$ cases had the T790M mutation, respectively. The T790M mutation rate between the wild-type and the heterozygous genotype of rs1050171, and the frequencies of the wild-type and heterozygous genotypes of rs1050171 in samples with the T790M mutation, are consistent with those of the total cases. The deviation of samples that were variant homozygous for rs1050171 may result from the
A

$\frac{220}{210} \stackrel{220}{T_{C A C C G T G C A A C T}}$

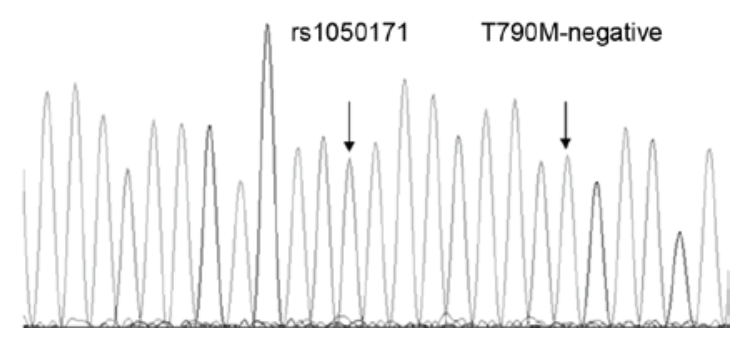

B

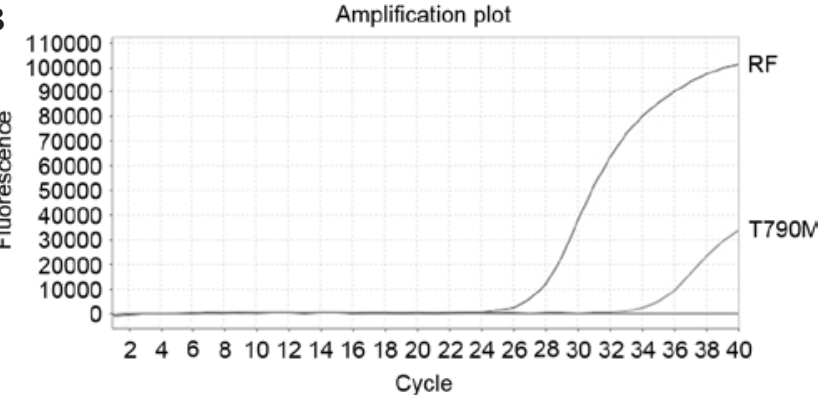

C

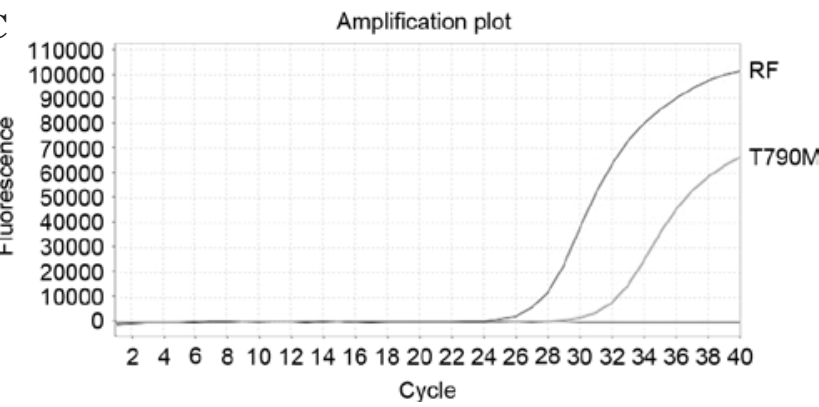

Figure 4. T790M detection results of sample N094 by different methods. (A) The sample N094 was detected as T790M-negative by sequencing, and is homozygous mutant genotype of rs1050171. The nucleotide location of the single nucleotide polymorphism is indicated by the arrows $(\downarrow)$ ). (B) The sample N094 was identified as T790M-negative by the ARMS-qPCR system 1 ( $\Delta$ Cq value $>7.5)$. (C) The sample N094 was demonstrated to have the T790M mutation by the ARMS-qPCR system 3 ( $\Delta$ Cq value $<7.5$ ). RF, reference; ARMS, amplification refractory mutation system; qPCR, quantitative polymerase chain reaction.

inadequate cases, since there was only one sample with the T790M mutation that was variant homozygous for rs 1050171 . No obvious association appears to exist between the mutation frequencies of T790M and rs1050171.

As for the influence of other two SNPs (rs148188503 and c. T2375C, p. L792P) on the ARMS-based T790M mutation assay, rs 148188503 was observed to be located 14 bp prior to T790M, which was farther away from T790M and had a lower mutation rate $(0.15 \%)$ compared with rs1050171. The novel SNP (c. T2375C, p. L792P) is located 6 bp beyond T790M, which is in the design range of the reverse ARMS primer, and has a low mutation rate $(0.15 \%)$. Since no high frequency SNPs located beyond T790M were detected in the present study, another possible solution is designing ARMS primers according to the other strand of the template. Further investigation about the influence of these two SNPs on ARMS-based T790M mutation assays is still required.

According to the ARMS principle, the last $3 \mathrm{bp}$ in the $3^{\prime}$ end of the ARMS primers are very important for the correct recognition and binding of the primer to the template (26-28). As a 
general rule, the mismatched bases are often designed in the last $3 \mathrm{bp}$ of the ARMS primer to discriminate between the wild-type and the mutant alleles (26-28). Notably, in the present study, the rs1050171 was observed to be located in the eighth base from the $3^{\prime}$ end of the ARMS primer, and the results revealed that it really affected the sensitivity of T790M mutation detection, particularly in samples with low mutation rates. It is possible that a similar situation may occur in other EGFR mutations and in point mutation in other genes, including $K R A S$ c.34G $>\mathrm{T}$ (G12C), BRAF c.1799T $>$ A (V600E) and PIK3CA c.1633G $>$ A (E545K). The present study highlighted the risk associated with the target neighboring SNPs (as far as 8 bp away from T790M mutation site in the current study), which may influence the effective detection of the target site, and should be considered when detecting novel point mutations.

In conclusion, the existence of T790M neighboring rs1050171 (located at $8 \mathrm{bp}$ prior to T790M) reduces the sensitivity of the ARMS-based T790M mutation detection assay and produces a $14.3 \%$ false-negative rate. The influence of target neighboring SNPs on the effective detection of the target mutation must be taken into consideration when starting a novel point mutation detection project.

\section{Acknowledgements}

The authors would like to thank Dr Congli Cai, Mr. Zhe Zhang and Mrs. Liqiong Li, researchers at Wuhan YZY Medical Science and Technology Co. Ltd. (Wuhan, China), for their advice and suggestions in developing the ARMS-based T790M mutation assay and for the construction of the plasmid samples.

\section{References}

1. Wang J, Ramakrishnan R, Tang Z, Fan W, Kluge A, Dowlati A, Jones RC and Ma PC: Quantifying EGFR alterations in the lung cancer genome with nanofluidic digital PCR arrays. Clini Chem 56: 623-632, 2010

2. Kim Y, Ko J, Cui Z, Abolhoda A, Ahn JS, Ou SH, Ahn MJ and Park K: The EGFR T790M mutation in acquired resistance to an irreversible second-generation EGFR inhibitor. Mol Cancer Ther 11: 784-791, 2012.

3. Kobayashi S, Boggon TJ, Dayaram T, Jänne PA, Kocher O, Meyerson M, Johnson BE, Eck MJ, Tenen DG and Halmos B EGFR mutation and resistance of non-small-cell lung cancer to gefitinib. N Engl J Med 352: 786-792, 2005.

4. Kuang Y, Rogers A, Yeap BY, Wang L, Makrigiorgos M, Vetrand K, Thiede S, Distel RJ and Jänne PA: Noninvasive detection of EGFR T790M in gefitinib or erlotinib resistant non-small cell lung cancer. Clinical cancer research: An official journal of the American Association for Cancer Research 15: 2630-2636, 2009.

5. Sun JM, Ahn MJ, Choi YL, Ahn JS and Park K: Clinical implications of T790M mutation in patients with acquired resistance to EGFR tyrosine kinase inhibitors. Lung Cancer 82: 294-298, 2013.

6. Yamada T, Azuma K, Muta E, Kim J, Sugawara S, Zhang GL, Matsueda S, Kasama-Kawaguchi Y, Yamashita Y, Yamashita T, et al: EGFR T790M mutation as a possible target for immunotherapy; identification of HLA-A*0201-restricted T cell epitopes derived from the EGFR T790M mutation. PloS One 8: e78389, 2013.

7. Denis MG, Vallée A and Théoleyre S: EGFR T790M resistance mutation in non small-cell lung carcinoma. Clin Chim Acta 444: $81-85,2015$.

8. Lin L and Bivona TG: Mechanisms of resistance to epidermal growth factor receptor inhibitors and novel therapeutic strategies to overcome resistance in NSCLC patients. Chemother Res Pract 2012: 817297, 2012.
9. Zhou W, Ercan D, Chen L, Yun CH, Li D, Capelletti M, Cortot AB, Chirieac L, Iacob RE, Padera R, et al: Novel mutant-selective EGFR kinase inhibitors against EGFR T790M. Nature 462: 1070-1074, 2009.

10. Yung TK, Chan KC, Mok TS, Tong J, To KF and Lo YM: Single-molecule detection of epidermal growth factor receptor mutations in plasma by microfluidics digital PCR in non-small cell lung cancer patients. Clin Cancer Res 15: 2076-2084, 2009.

11. Thomas RK, Nickerson E, Simons JF, Jänne PA, Tengs T, Yuza Y, Garraway LA, LaFramboise T, Lee JC, Shah K, et al: Sensitive mutation detection in heterogeneous cancer specimens by massively parallel picoliter reactor sequencing. Nat Med 12: 852-855, 2006.

12. Guha M, Castellanos-Rizaldos E and Makrigiorgos GM: DISSECT method using PNA-LNA clamp improves detection of T790M mutation. PloS One 8: e67782, 2013.

13. Bai H, Wang Z, Wang Y, Zhuo M, Zhou Q, Duan J, Yang L, Wu M, An T, Zhao J and Wang J: Detection and clinical significance of intratumoral EGFR mutational heterogeneity in Chinese patients with advanced non-small cell lung cancer. PloS One 8: e54170, 2013.

14. Board RE, Ellison G, Orr MC, Kemsley KR, McWalter G, Blockley LY, Dearden SP, Morris C, Ranson M, Cantarini MV, et al: Detection of BRAF mutations in the tumour and serum of patients enrolled in the AZD6244 (ARRY-142886) advanced melanoma phase II study. Br J Cancer 101: 1724-1730, 2009.

15. Chu H, Zhong C, Xue G, Liang X, Wang J, Liu Y, Zhao S, Zhou Q and Bi J: Direct sequencing and amplification refractory mutation system for epidermal growth factor receptor mutations in patients with non-small cell lung cancer. Oncol Rep 30: 2311-2315, 2013.

16. Ellison G, Donald E, McWalter G, Knight L, Fletcher L, Sherwood J, Cantarini M, Orr M and Speake G: A comparison of ARMS and DNA sequencing for mutation analysis in clinical biopsy samples. J Exp Clin Cancer Res 29: 132, 2010.

17. Franklin WA, Haney J, Sugita M, Bemis L, Jimeno A and Messersmith WA: KRAS mutation: Comparison of testing methods and tissue sampling techniques in colon cancer. J Mol Diagn 12: 43-50, 2010.

18. Fukuoka M, Wu YL, Thongprasert S, Sunpaweravong P, Leong SS, Sriuranpong V, Chao TY, Nakagawa K, Chu DT, Saijo N, et al: Biomarker analyses and final overall survival results from a phase III, randomized, open-label, first-line study of gefitinib versus carboplatin/paclitaxel in clinically selected patients with advanced non-small-cell lung cancer in Asia (IPASS). J Clin Oncol 29: 2866-2874, 2011.

19. Hamfjord J, Stangeland AM, Skrede ML, Tveit KM, Ikdahl T and Kure EH: Wobble-enhanced ARMS method for detection of KRAS and BRAF mutations. Diagn Mol Pathol 20: 158-165, 2011.

20. Harlé A, Lion M, Lozano N, Husson M, Harter V, Genin P and Merlin JL: Analysis of PIK3CA exon 9 and 20 mutations in breast cancers using PCR-HRM and PCR-ARMS: Correlation with clinicopathological criteria. Oncol Rep 29: 1043-1052, 2013.

21. Huang T, Zhuge J and Zhang WW: Sensitive detection of BRAF V600E mutation by amplification refractory mutation system (ARMS)-PCR. Biomark Res 1: 3, 2013.

22. Liu Y,Liu B, Li XY, Li JJ, Qin HF, Tang CH, Guo WF, Hu HX, Li S, Chen CJ, et al: A comparison of ARMS and direct sequencing for EGFR mutation analysis and tyrosine kinase inhibitors treatment prediction in body fluid samples of non-small-cell lung cancer patients. J Exp Clin Cancer Res 30: 111, 2011.

23. Machnicki MM, Glodkowska-Mrowka E, Lewandowski T, Ploski R, Wlodarski P and Stoklosa T: ARMS-PCR for detection of BRAF V600E hotspot mutation in comparison with real-time PCR-based techniques. Acta Biochim Pol 60: 57-64, 2013.

24. Ogasawara N, Bando H, Kawamoto Y, Yoshino T, Tsuchihara K, Ohtsu A and Esumi H: Feasibility and robustness of amplification refractory mutation system (ARMS)-based KRAS testing using clinically available formalin-fixed, paraffin-embedded samples of colorectal cancers. Jpn J Clin Oncol 41: 52-56, 2011.

25. Zhang B, Xu CW, Shao Y, Wang HT, Wu YF, Song YY, Li XB, Zhang Z, Wang WJ, Li LQ and Cai CL: Comparison of droplet digital PCR and conventional quantitative PCR for measuring gene mutation. Exp Ther Med 9: 1383-1388, 2015.

26. Pettersson M, Bylund M and Alderborn A: Molecular haplotype determination using allele-specific PCR and pyrosequencing technology. Genomics 82: 390-396, 2003

27. Vlassov VV, Laktionov PP and Rykova EY: Circulating nucleic acids as a potential source for cancer biomarkers. Curr Mol Med 10: 142-165, 2010.

28. Ye S, Dhillon S, Ke X, Collins AR and Day IN: An efficient procedure for genotyping single nucleotide polymorphisms. Nucleic Acids Res 29: E88-E88, 2001. 